\title{
Prevalência de queixa álgica musculoesquelética em trabalhadores de empresa de bijuteria
}

\author{
Prevalence of musculoskeletal pain in jewelry company workers
}

Tamiris Augusta de Noronha, ${ }^{1}$ Hélcio Balbino dos Santos, ${ }^{2}$ Kelly Christina de Faria, ${ }^{1}$

César Augusto França Abraão, ${ }^{3}$ Gisélia Gonçalves de Castro ${ }^{1}$

'Centro Universitário do Cerrado (Unicerp), Patrocínio, MG, Brasil.

¿Centro Universitário do Planalto de Araxá (Uniaraxá), Araxá, MG, Brasil.

3Universidade Federal do Triângulo Mineiro (UFTM), Uberaba, MG, Brasil.

Recebido em: 13/08/2016 / Aceito em: 08/09/2016 / Publicado em: 18/10/2016

helciosantos@uniaraxa.edu.br

\section{RESUMO}

O setor de bijuteria é crescente, nos últimos três anos, com media de $10 \%$ ao ano. Os trabalhadores desta área empresarial, respeitando claro os diversos setores, estão sujeitos a inúmeros distúrbios musculoesqueléticos, podendo acometer diversas áreas do corpo, principalmente, quando os trabalhadores se encontram expostos a fatores de risco como posturas inadequadas, repetitividade, uso de força excessiva e a exposição a vibrações. Objetivo: identificar a prevalência de queixa álgica musculoesquelética em trabalhadores de empresa de bijuteria. Método: estudo de natureza quantitativa, observacional analítica, com pesquisa de campo com a aplicação de questionário sócio demográfico e o diagrama de Corlett. Participaram do presente estudo 30 trabalhadores (24 mulheres e 6 homens) de uma empresa de bijuteria situada no interior do estado de Minas Gerais. Resultados: mostraram que $86,6 \%$ dos funcionários relata algum quadro de dor como decorrência do trabalho repetitivo, com predominância do sexo feminino. Os resultados evidenciaram que no setor de colagem, a postura de trabalho sentada, a jornada de trabalho a partir de 9 horas diárias, o período final da jornada de trabalho relacionou com maior número de pontos de dor e ao parâmetro intensidade, sobremaneira nas regiões da coluna cervical e lombar. Há um pequeno índice de procura ao serviço médico e baixa taxa de automedicação. Considerações finais: os resultados denotam uma relação entre sexo feminino, idade, postura de trabalho, tipo de trabalho, número de horas trabalhadas, áreas corporais, intensidade e período do dia com a dor musculoesquelética em trabalhadores do setor de bijuterias. Ainda que existam achados sintomatológicos sugestivos desta relação, há uma diversidade metodológica e pequeno número de estudos que avaliam a dor neste grupo de trabalhadores.
Palavras-chave: Saúde ocupacional; Indústria de bijuterias; Dor musculoesquelética

\section{ABSTRACT}

The jewelry industry is growing, the last three years with an average of $10 \%$ per year. The workers of this business area, respecting of course the various sectors, are subjected to countless musculoskeletal disorders and can affect many areas of the body, especially when workers are exposed to risk factors such as inadequate posture, repetitivity, excessive use of force and exposure the vibrations. Objetive: identify the prevalence of musculoskeletal pain complaints in jewelry company employees. Method: study, analytical observational field research with the application of demographic questionnaire and Corlett diagram. The study included 30 employees 124 women and 6 men) of a jewelry company located in the state of Minas Gerais. Results: showed that $86.6 \%$ of employees reported some pain framework due to repetitive work, especially women. The results showed that the bonding industry, sitting working posture, working hours from 9 hours per day, the final period of working hours were associated with greater number of pain points and parameter intensity greatly in regions cervical and lumbar spine. There is a low rate of demand for medical service and low self-medication rate. Closing remarks: the results show a relation between female gender, age, working posture, type of work, hours worked, body areas, intensity and time of day with musculoskeletal pain parameter workers jewelry sector. Though there symptomatology suggestive findings of this relationship, there is a methodological diversity and small number of studies that evaluated pain in this group of workers.

Keywords: Occupational Health; Jewelry Industry; Skeletal Muscle Pain. 


\section{INTRODUÇÃO}

Indubitavelmente a temática Saúde do Trabalhador, após o advento da Revolução Industrial, tem sido foco de muitos estudos, tanto pelas questões econômicas, como pelos debates inerentes ao processo saúde-doença ocupacional. A sociedade industrializada, através do uso de tecnologias e equipamentos avançados, requer ao trabalhador uma mão de obra automatizada e repetitiva que, associada ao mercado de trabalho intenso, competitivo e com altas pressões, pode levar esse trabalhador a desenvolver entre outros sintomas, alguns distúrbios osteomusculares. ${ }^{1}$ Associada à atividade laboral intensa, no cenário atual existe uma tendência do trabalhador se tornar sedentário pela falta de tempo, para cuidar da sua saúde e praticar algum tipo de atividade física, repercutindo nas condições biopsicossoais do trabalhador. ${ }^{2}$

Há um duplo papel do trabalho, pois além de proporcionar o sustento e uma melhor qualidade de vida e para promover o desenvolvimento dos indivíduos e favorecer a valorização e auto- realização, quando realizado sob condições e ambientes inadequados, pode prejudicar a saúde, causar doenças e a levar à morte. ${ }^{3}$

A saúde do trabalhador está diretamente ligada a fatores econômicos, tecnológicos, sociais e organizacionais associados ao perfil de produção e consumo, além dos fatores de risco de natureza física, química, mecânica, biológica e ergonômica, que se encontram nos processos produtivos. ${ }^{4}$ Os distúrbios osteomusculares relacionados ao trabalho (DORT) vinculam-se diretamente à realização de atividades ocupacionais e às condições de trabalho.

As lesões por esforço repetitivo (LER) são manifestações relativamente comuns do sistema musculoesquelético em trabalhadores associadas à presença de vários sintomas tais como a fadiga, dor, parestesia e sensação de peso, de forma lenta ou progressiva. ${ }^{5}$

No geral, as patologias causadas pelas LER/DORT não são de difícil tratamento, o que, em muitos casos, não se aplica a condições crônicas, por exemplo. No entanto, pode gerar quadro álgico, perda da função e edema dentre outros sinais e sintomas. São responsáveis por uma importante e significativa parcela de incapacidade funcional, absenteísmo e decréscimo da modernidade, bem como de aumento da encargos previdenciários. ${ }^{6}$

Atividades laborais executadas em linhas de produção segmentadas, em que o trabalhador exerce uma atividade repetitiva e por longos períodos, associadas a cargas tensionais, são fontes potenciais etiológicas a serem investigadas na gênese dos distúrbios musculoesqueléticos. ${ }^{7}$

Neste contexto, os trabalhadores do setor de joias e bijuterias constituem um público vulnerável ao desenvolvimento de LER/DORT, uma vez que a linha de produção das mesmas exige esforços e cargas sustentadas, em ambientes muitas vezes não ergonômicos. ${ }^{8}$ Na fabricação de joias predomina a utilização do trabaIho manual, centrado em atividade repetitiva, sobretudo nos processos de montagem, soldagem e colagem. ${ }^{9}$

Neste sentido, identificar o perfil álgico destes trabalhadores é uma forma inicial de identificar possíveis fatores etiológicos, de necessidades de mudanças laborais que aperfeiçoem a produção, mas principalmente na criação de um ambiente laboral saudável, ou seja, é uma questão básica de se pensar e de se agir em relação à promoção da saúde dos trabalhadores do setor de joias e bijuterias. Desta forma, o presente estudo teve como objetivo identificar o perfil álgico dos trabalhadores de uma indústria de bijuterias no interior de Minas Gerais, enquanto estratégia de promoção de saúde, dentro da perspectiva da criação de ambientes favoráveis à saúde, preconizada pela carta de Otawa. ${ }^{10}$

\section{MÉTODO}

Tratou-se de um estudo de natureza quantitativa, transversal, observacional analítica com pesquisa de campo. O cenário da pesquisa foi à cidade de Patrocínio, município localizado na região do Alto Paranaíba no oeste do estado de Minas Gerais, que possui uma extensão territorial de $2.867,39 \mathrm{~km}^{2}$ e população total de 86.467 habitantes. Os dados foram coletados na Empresa Flávia Caldeira, especializada na fabricação e vendas de bijuterias no comércio de atacado e varejo, com atuação nacional, mediante consentimento prévio, através da assinatura de um termo de autorização.

A pesquisa foi composta por 30 participantes (24 mulheres e 6 homens) dos setores de criação e design, montagem e colagem das peças, fundição do metal bruto, qualidade da produção, administrativo e vendas atacado e varejo. Foram exclusos do estudo, os funcionários que não trabalhavam diretamente com a montagem das bijuterias e/ou que não permanecessem na mesma posição por longos períodos, neste caso, os setores de cobrança e limpeza.

A coleta de dados foi realizada por meio de dois instrumentos. Para caracterização dos participantes foi utilizado um questionário semi-estruturado elaborado pela equipe. Este questionário foi subdividido em três partes: a primeira para caracterização dos participantes; a segunda acerca das informações relativas ao trabalho e a terceira, sobre as características da dor.

Para a identificação do local da dor e desconforto auto-declarado foi aplicado o diagrama de Corllet, Wilson e Manenica. ${ }^{11}$ Foram analisadas as seguintes variáveis: sexo, idade, tempo de trabalho, setor de trabalho, postura de maior prevalência e local do quadro álgico. A coleta de dados foi efetivada no mês de setembro de 2014. Os dados foram avaliados através da estatística descritiva, apresentados em percentuais, média e desvio padrão e demonstrado em gráficos e tabelas. A pesquisa foi submetida ao Comitê de Ética CEP-UNICERP, com aprovação sob protocolo $n^{\circ} 20131450 F I S 04$, respeitando os aspectos éticos em pesquisas com seres humanos.

Cada participante assinou um Termo de Consentimento Livre e Esclarecido (TCLE), no qual eram informados os objetivos, justificativa, procedimentos de coleta de dados e fins acadêmicos, bem como foram respeitados todos os aspectos éticos determinados pela Resolução 466/12, do Conselho Nacional de Saúde, resguardando os direitos éticos em pesquisas com seres humanos.

\section{RESULTADOS}

Dos 30 participantes da pesquisa, 24 (80\%) são representados pelo sexo feminino e 6 representados 
pelo sexo masculino (20\%), 26 trabalhadores $(86,6 \%)$ sentem algum tipo de dor. No que se refere à idade dos funcionários, houve uma amostra diversificada, tendo uma variação de 16 a 59 anos. A média de idade no sexo feminino foi de $28,04 \pm 10,78$ anos; enquanto no masculino $24,83 \pm 4,31$ anos.

Para análise dos dados, todos os participantes da pesquisa que compõem o quadro funcional da fábrica de bijuteria pesquisada foram agrupados em setores, sendo que 12 (40\%), a maioria encontrou-se no setor de colagem; enquanto $5(16,6 \%)$ no setor de fundição, $4(13,3 \%)$ no setor de montagem, $6(20 \%)$ nos setores de vendas, estoque e designer ( $2-6,6 \%$ em cada setor), finalizando o quadro de funcionários com o setor de criação, administrativo e qualidade com 1 funcionário em cada um destes setores (3,3\%, cada).

Em relação ao tempo de trabalho observamos que $10(33,3 \%)$ funcionários possuem um tempo inferior a 12 meses de trabalho, $40 \%$ representando dos funcionários, com tempo de 1 a 5 anos de trabalho, 6 (20\%) de 6 a 10 anos e $2(6,6 \%)$ estão na empresa há 11 anos ou mais.

Quanto à jornada de trabalho, observou-se que $11(36,6 \%)$ funcionários trabalham 8 horas diárias, 14 $(46,6 \% \%) 9$ horas por dia, $3(9,9 \%) 10$ horas diárias e $2(6,6 \%) 12$ horas por dia. É importante ressaltar que, além da jornada de trabalho 18 (60\%) funcionários afirmaram realizar horas extras. Questionados quanto à realização de outros trabalhos fora do horário na empresa, apenas dois indivíduos o fazem, sendo que um trabalha com salão de beleza $(3,3 \%)$ e outro com artesanato $(3,3 \%)$.

Analisando as posturas mantidas durante a jornada de trabalho, 23 funcionários $(76,7 \%)$ da amostra se mantém sentada, durante a jornada de trabalho, e 7 $(23,3 \%)$ de pé.

Os participantes relataram 104 queixas álgicas, ressaltando que muitos têm múltiplas regiões de dor. Os principais segmentos com queixas de dor, considerando todos os setores de trabalho foram o pescoço e as costas com 20 relatos $(19,2 \%), 13(12,5 \%)$ ombros, 8 $(7,7 \%)$ mencionam os punhos, $9(8,6 \%)$ dores nas pernas, $6(5,8 \%)$ dores nas mãos, $5(4,8 \%)$ descrevem os braços, a região do quadril e a lombar, $3(2,9 \%)$ relatam dor no antebraço e $2(1,9 \%)$ no tornozelo.

Ao analisar por setor, os participantes que trabaIham na colagem apresentaram maior quadro álgico, seguidos pelos trabalhadores das áreas de montagem, fundição, estoque e designer. Com um total de 86 $(82,7 \%$ das 104 queixas) de relato de dor foram encontrados nos setores, o membro mais acometido pelas dores e desconfortos foi o membro superior com 70 relatos $(67,3 \%)$; já, os membros inferiores representaram 16 relatos $(15,3 \%)$.

Em relação à região mais acometida, observa-se o pescoço com 19 queixas (18,3\%) seguido pelas queixas de dor nas costas que totalizaram $16(15,4 \%)$, a dor ombro $13(12,5 \%)$, e apenas $3(2,9 \%)$ da amostra alegaram sentir dores no antebraço. Já o membro inferior teve $16(15,3 \%)$ relatos e o segmento mais acometido foram as pernas com 9 casos representando $(8,6 \%), 5(4,8 \%)$ de dores no quadril e lombar e 2 $(1,9 \%)$ de dor no tornozelo (Tabela 1 ).

No setor de vendas, qualidade, criação e administrativo, houve um total de 18 relatos de locais dolorosos (17,3\% das 104 queixas totais). Nesses setores o membro superior também foi o mais atingido. Os segmentos mais acometidos foram o pescoço, as costas e o punho com 3 relatos cada, representando 2,9\% em cada região anatômica, 1 (0,96\% para cada menção à área de dor) mencionou dores no ombro, no braço, antebraço e na mão. Os membros inferiores com 5 relatos sendo a região do quadril e lombar e as pernas mais atingidos com 2 relatos $(1,92 \%)$ cada, e $1(0,96 \%)$ de dores no tornozelo (Tabela 2$)$.

Em relação à intensidade da dor ou desconforto que sentem no dia-a-dia, $2(6,7 \%)$ relatam uma dor ou desconforto insuportável, 2 descrevem ser muito grave $(6,7 \%), 3(10 \%)$ citam uma dor grave, $11(36,7 \%)$ moderada, $8(26,6 \%)$ dor leve e $4(13,3 \%)$ não sentem nenhuma dor ou desconforto.

Em relação ao período do dia em que os sujeitos são acometidos de dores e desconfortos, $2(6,7 \%)$ mencionaram sentir dores do período da manha, 21 (70\%) no período da tarde, $6(20 \%)$ à noite e 1 (3,3\%) de madrugada.

Apesar das queixas dolorosas, uma pequena quantidade de funcionários relata que já buscou auxílio médico. Do total, 8 (27\%) relatam ter recorrido a algum profissional da saúde para alívio das dores, 22 (73\%) não buscaram auxílio.

Dentro desse contexto verificou que a automedicação foi referida por 7 (23\%) que alegaram tomar medicamentos, quando sentem muitas dores, e 23 (77\%) afirmaram que não se automedicam.

Tabela 1 - Relação dos principais setores de trabalho e as regiões do corpo com quadro álgico (colagem, montagem, fundição, estoque e designer).

\begin{tabular}{|c|c|c|c|c|c|}
\hline \multirow[b]{2}{*}{ Queixa Principal } & \multicolumn{4}{|c|}{ Local } & \multirow[b]{2}{*}{ Tota } \\
\hline & $\begin{array}{l}\text { Membros } \\
\text { superiores }\end{array}$ & $\%$ & $\begin{array}{l}\text { Membros } \\
\text { inferiores }\end{array}$ & $\%$ & \\
\hline Dor no pescoço & 19 & $18,3 \%$ & - & - & 19 \\
\hline Dor nas costas & 16 & $15,4 \%$ & - & - & 16 \\
\hline Dor no ombro & 13 & $12,5 \%$ & - & - & 13 \\
\hline Dor no punho & 8 & $7,7 \%$ & - & - & 8 \\
\hline Dor na mão & 6 & $5,8 \%$ & - & - & 6 \\
\hline Dor no braço & 5 & $4,8 \%$ & - & - & 5 \\
\hline Dor no antebraço & 3 & $2,9 \%$ & - & - & 3 \\
\hline Dor na perna & - & - & 9 & $8,6 \%$ & 9 \\
\hline Dor no quadril e lombar & - & - & 5 & $4,8 \%$ & 5 \\
\hline Dor no tornozelo & - & - & 2 & $1,9 \%$ & 2 \\
\hline Total & 70 & - & 16 & - & 86 \\
\hline
\end{tabular}


Tabela 2 - Relação dos setores de trabalho e as regiões do corpo com quadro álgico (vendas, criação, qualidade e administrativo).

\begin{tabular}{|c|c|c|c|c|c|}
\hline \multirow[b]{2}{*}{ Queixa Principal } & \multicolumn{4}{|c|}{ Local } & \multirow[b]{2}{*}{ Total } \\
\hline & $\begin{array}{l}\text { Membros } \\
\text { superiores }\end{array}$ & $\%$ & $\begin{array}{l}\text { Membros } \\
\text { inferiores }\end{array}$ & $\%$ & \\
\hline Dor no pescoço & 3 & $2,9 \%$ & - & - & 3 \\
\hline Dor nas costas & 3 & $2,9 \%$ & - & - & 3 \\
\hline Dor no ombro & 3 & $2,9 \%$ & - & - & 3 \\
\hline Dor no punho & 1 & $0,96 \%$ & - & - & 1 \\
\hline Dor na mão & 1 & $0,96 \%$ & - & - & 1 \\
\hline Dor no braço & 1 & $0,96 \%$ & - & - & 1 \\
\hline Dor no antebraço & 1 & $0,96 \%$ & - & - & 1 \\
\hline Dor na perna & - & - & 2 & $1,92 \%$ & 2 \\
\hline Dor no quadril e lombar & - & - & 2 & $19,2 \%$ & 2 \\
\hline Dor no tornozelo & - & - & 1 & $0,96 \%$ & 1 \\
\hline Total & 13 & - & 5 & - & 18 \\
\hline
\end{tabular}

\section{DISCUSSÃO}

Ao se abordar a questão da dor, deve-se entendê-la como uma condição multifatorial e visto como resultado não apenas de um quadro de lesão tecidual, mas numa possível ameaça ao organismo. ${ }^{12}$ No caso dos trabaIhadores ligados ao setor de bijuteria, esta condição também se aplica uma vez que parâmetros intrínsecos (sexo, idade, doenças pregressas, fatores psicossociais dentre outros) e extrínsecos (ergonomia, organização, atividade a ser executada, horas trabalhadas, outros empregos concomitantes, etc.) influenciam na gênese e no controle da mesma. ${ }^{13}$

No presente estudo, observou-se um espectro amostral muito variável em relação ao parâmetro idade. Este é um fator, dentre outros, que de acordo com alguns trabalhos é positivamente correlacionado com a dor, sobretudo pelo déficit de oxigenação tecidual e a redução na condição de hidratação articular em pessoas mais velhas. ${ }^{14}$

Houve uma maior expressão do sexo feminino nesta pesquisa. Os distúrbios musculoesqueléticos acometem mais as mulheres que os homens, dados que podem ser explicados pelo fato de que as mulheres estão presentes nas tarefas repetitivas e monótonas e, ainda a diferença de massa muscular e composição corporal, podem representar um fator de risco predisponente de sintomatologia dolorosa. ${ }^{15}$

As especificidades da atividade laboral e o tempo de execução da mesma também são parâmetros a serem considerados na investigação do quadro álgico. Observou-se no presente estudo que $46,6 \%$ dos participantes exercem cerca de 9 horas diárias de trabaIho. Levando em conta o tipo de trabalho realizado na empresa, ${ }^{16}$ considera como fator de risco, a obrigação em manter um ritmo de trabalho acelerado devido a incentivos salariais por maiores produtividades, trabaIho repetitivo sem períodos de pausas para descanso, jornadas prolongadas de trabalho, realizações de horas extras e o número insuficiente de funcionários.

O fator carga horária associada ao tipo de atividade executada pode promover inúmeras adaptações cinético-funcionais que podem ser entendidas pelo próprio corpo, como benéficas à realização da tarefa la- boral, mas que culminem em desequilíbrios musculares (força e flexibilidade) e adaptações posturais traduzidos fisicamente como dor. ${ }^{17}$

Outro fator importante nesta investigação da dor desta população, é a questão da postura de trabalho e o tipo de atividade executada. Observou-se, na presente pesquisa, que indivíduos que trabalham mais tempo sentados (23 - cerca de $76,7 \%$ ) e no setor de colagem (12 - 40\%) sentem mais dor, tanto nos parâmetros de intensidade, quanto na quantidade de locais de dor avaliados, através do diagrama de Courlet. Na função de vendedor, por exemplo, houve um número menor de relatos de dor comparado ao setor de colagem, visto que nesta função ocorrem mudanças posturais com maior frequência, de sentado para de pé, bem como uma movimentação maior do corpo.

Múltiplos fatores podem estar relacionados a estes números. A manutenção prolongada na postura sentada aumenta a sobrecarga axial na coluna vertebral, favorecendo a desidratação discal; dificulta a retirada de metabólitos e se, associada aos movimentos repetitivos dos membros superiores, levando a quadros de hipóxia tecidual, fadiga periférica, insuficiência muscular e claro, dor. ${ }^{18}$

Os resultados do presente estudo corroboram com estas observações uma vez que trabalhar sentado por longos períodos foi responsável por cerca de 82,7\% das queixas álgicas. Mais uma vez, o setor de colagem apresentou os dados mais expressivos na intensidade e números de áreas de dor.

Nas atividades que exigem uma boa visualização, assim como o setor de montagem e colagem, existe a tendência de aproximar à cabeça e fletir a coluna cervical, a fim de aproximar os olhos do produto a ser fabricado, gerando uma tensão no local. ${ }^{19}$ Tendo em vista que o processo colagem e montagem de bijuteria são realizados na posição sentada, dois autores relatam que a permanência na postura sentada por até seis horas com pequenos intervalos em pé, podem causar dores, fadiga e até mesmo alterações posturais. ${ }^{20}$ A flexão também contínua da coluna lombar, sobremaneira associada aos movimentos torcionais e rotacionais, também gera um aumento da pressão. Estes achados também reforçam a relação da postura sentada com os locais de dor, já que 
quadros álgicos mais intensos foram observados nos trabalhadores, que exercem sua atividade na posição sentada, com maiores queixas nas regiões de pescoço e costas $(38,4 \%$ das queixas totais).

Corroborando com esse estudo, ${ }^{21}$ dores nos membros superiores ocorrem quando se trabalha por muito tempo sem apoio e isso ocorre, principalmente, com o uso de ferramentas manuais, agravando ainda, quando são realizados movimentos repetitivos com as mãos ou quando há aplicação de força, como é comum em muitas das atividades laborais dos trabalhadores do setor de bijuteria.

Em contrapartida, em outras funções, como no caso do vendedor, por exemplo, houve um número menor de relatos de dor comparado ao setor de colagem, visto que nesta função ocorrem mudanças posturais com maior frequência, de sentado para de pé, bem como uma movimentação maior do corpo.

Indubitavelmente, a dor é o sintoma mais descrito por estes profissionais, podendo oscilar seus efeitos entre leves, moderados, chegando a níveis intensos, o que compromete a qualidade de vida das pessoas. No presente estudo $11(36,7 \%)$ funcionários sentem dores moderadas, principalmente observadas ao final do dia de trabalho, relacionado, provavelmente, à fadiga do sistema musculoesquelético, após a execução de uma mesma função por um longo período, exigindo demasiadamente uma determinada estrutura corporal. ${ }^{7}$

Apesar da manifestação quanto a alta ocorrência de sintomas musculoesqueléticos, na população estudada, $27 \%$ relatam ter procurado algum profissional da área da saúde por causa desses sintomas. Esses resultados evidenciam que a população estudada está comprometida pelos sintomas musculoesqueléticos, apesar de não ser possível afirmar que esses trabalhadores possuem LER/DORT, pela complexidade no diagnostico desses desgastes.

Outro fator a ser abordado dentro do questionário é a automedicação; ${ }^{22}$ o trabalhador aprende a conviver com a dor e se automedicam, ou seja, faz o uso de medicação sem prescrição médica. Uma amostra de $7(23 \%)$ sujeitos relatou fazer o uso de medicamentos analgésicos antes e durante a jornada de trabalho, amenizando as dores para que consigam realizar suas funções e, após a jornada, em seu momento de descanso, a fim de aliviar os sintomas para que possam dormir.

O que se observa é que existe sim um número expressivo de participantes que sentem e convivem com a dor. Todavia, ainda que exista uma relação entre distúrbios musculoesqueléticos, fatores de risco ergonômicos e efeitos metabólicos e fisiológicos, esta relação não está bem estabelecida, já que pode ser e/ou sofre inúmeras influências, fato que dificulta assertividade entre estes fatores e a dor na população estudada.

\section{CONSIDERAÇÕES FINAIS}

Conclui-se com o estudo que a dor musculoesquelética está presente, quase que na totalidade dos funcionários, sobretudo em nível moderado de intensidade. Alguns setores laborais que envolvam atividades repetitivas de membros superiores, postura sentada e jornada de trabalho prolongada ( $\geq 9$ horas diárias), como o setor de colagem, por exemplo, apresentam índices maiores de dor, mormente no pescoço e região de coluna lombar, principalmente ao final da jornada de trabalho. Os resultados evidenciam que ainda que seja encontrado um nível considerado de queixas álgicas, a maioria dos participantes não busca ajuda médica e não faz automedicação.

A avaliação da dor musculoesquelética em trabalhadores do setor de bijuterias é uma condição que merece ser investigada. Todavia, entende-se como uma condição multifatorial; com uma diversidade de metodologias empregadas, em uma série não muito extensa de trabalhos sobre o tema. Desta forma, ainda que exista a sugestão de aspectos organizacionais, biomecânicos e psicossociais, tem-se uma certa dificuldade de estabelecer e compreender claramente a relação destes fatores com a dor.

\section{REFERÊNCIAS}

1. Roman-Liu D, Bugajska J, Tokarski T. Comparative Study of Upper Limb Load Assessment and Occurrence of Musculoskeletal Disorders at Repetitive Task Workstations. Ind Health 2014;52(6):461-470. doi: 10.2486/indhealth.2013-0232.

2. Dean $E$, Söderlund $A$. What is the role of lifestyle behaviour change associated with non-communicable disease risk in managing musculoskeletal health conditions with special reference to chronic pain? BMC Musculoskeletal Disorders 2015;16:87. doi: 10.1186/s12891-015-0545-y.

3. Sanches EM, Cutolo LRA, Soares P, Silva RM. Organização do trabalho, sintomatologia dolorosa e significado de ser portador de LER/DORT. Psicol. Argum 2010;28(63):313-324.

4. Fischer FM. Relevância dos fatores psicossociais do trabalho na saúde do trabalhador. Rev Saúde Pública 2012;46(3):401-6.

5. Alcântara MA, Nunes GS, Ferreira BCMS. Distúrbios osteomusculares relacionados ao trabalho: o perfil dos trabalhadores em benefício previdenciário em Diamantina (MG, Brasil). Ciênc. saúde coletiva 2011;16(8):3427-3436. doi: 10.1590/S1413-81232011000900010.

6. Macdonald W, Oakman J. Requirements for more effective prevention of work-related musculoskeletal disorders. BMC Musculoskelet Disord 2015;16:29. doi: 10.1186/s12891015-0750-8.

7. Salve UR. Prevalence of musculoskeletal discomfort among the workers engaged in jewelry manufacturing. Indian Journal of Occupational and Environmental Medicine 2015;19(1):4455. doi: 10.4103/0019-5278.157008.

8. Batiz EC, Nunes JIS, Olga Licea OEA. Prevalence of muscle-skeletal symptoms for load workers. Production 2013;23(1):168-177. doi: 10.1590/S010365132012005000030 .

9. Gonçalves JM, Camarotto, JA. Estratégias operatórias frente ao trabalho repetitivo. Production2015;25(1):190-200. doi: 10.1590/S0103-65132013005000087.

10. Carta de Ottawa, Primeira Conferência Internacional sobre Promoção da Saúde. Canadá, 1986.

11. Corlett N, Wilson J, Manenica I. The ergonomics of working postures. London: Taylor \& Francis, 1986.

12. Bonica JJ. The need of a taxonomy. Pain 1979;6(3):247-8. doi: 10.1016/0304-3959(79)90046-0

13. Mesquita CC. Musculoskeletal Disorders in Workersrisk factors: What Can We Do? Occup Med Health Aff 2013;1(3):1-4. doi: 10.4172/2329-6879.1000113

14. Kinge JM, Knudsen AK, Skirbekk V, Vollset SE. 
Musculoskeletal disorders in Norway: prevalence of chronicity and use of primary and specialist health care services. BMC Musculoskeletal Disorder 2015;16:75. doi: 10.1186/s12891 015-0536-z.

15. Choobineh A, Daneshmandi H, Tabatabaee SH. The Prevalence Rate of Work-Related Musculoskeletal Disorders Among Iranian Female Workers. Women's Health Bull 2015;2(4): e27334. doi: 10.17795/whb27334.

16. Epiphanio EB, Vilela JRPX. Perícias Médicas - Teoria e Prática. Rio de Janeiro: Guanabara Koogan, 2009. 394p

17. Delloiacono Nancy. Musculoskeletal Safety for Older Adults in the Workplace. Workplace Health Saf 2015;63(2):48-53. doi: $10.1177 / 2165079915570299$.

18. Akodu A, Akinfeleye A, Atanda L, Giwa S. Work-related musculoskeletal disorders of the upper extremity with reference to working posture of secretaries. South African Journal of Occupational Therapy 2015;45(3):16-22. doi: 10.17159/2310-3833/2015/v45n3/a4.

19. Renner Jacinta Sidegun. Prevenção de Distúrbios Osteomusculares Relacionadas ao Trabalho. Porto Alegre: Boletim da Saúde 2005;19(1):73-80. Disponível em: <http:// www.esp.rs.gov.br>. Acesso em: 04 out. 2014.

20. Rego ARON, Scartoni FR. Alterações Posturais de Alunos de 5 a 6 a séries do Ensino Fundamental. Fit Perf J 2008;7(1):105. doi: 103900/fpj.7.1.10.p.

21. Picoloto D, Silveira E. Prevalência de Sintomas Osteomusculares e Fatores Associados em Trabalhadores de uma Indústria Metalúrgica de Canoas - RS. Ciênc. Saúde Coletiva [online] 2008;3(2):507-516. doi: 10.1590/S141381232008000200026.

22. Aquino D S. Por que o uso racional de medicamentos deve ser uma prioridade? Ciênc. Saúde Coletiva [online] 2008;13(suppl):733-736. doi: 10.1590/S141381232008000700023.

Como citar: NORONHA, Tamiris Augusta de et al. Prevalência de queixa álgica musculoesquelética em trabalhadores de empresa de bijuteria. Cinergis, Santa Cruz do Sul, v. 18, n. 1, out. 2016. ISSN 2177-4005. Disponível em: <https://online. unisc.br/seer/index.php/cinergis/article/view/8112>. Acesso em: 11 out. 2016. doi:http://dx.doi.org/10.17058/cinergis. v17i3.8112. 